\title{
Primer reporte de Enterobacterales dobles productores de carbapenemasas en hospitales de Paraguay. Año 2021
}

\author{
Nancy Melgarejo Touchet ${ }^{1 D}{ }^{1}$, Sofía Busignani ${ }^{1}{ }^{1}$, Pamela Dunjo $^{1}$ (D), Mariel Brítez ${ }^{\text {iD }}{ }^{1}$, \\ Aníbal Kawabata ${ }^{2}$, Marlene Silvagni ${ }^{3}$, Carolina Rojas ${ }^{4}$, Eva Mereles 5 , Evelyn \\ López 6, Juana Salinas 7 , Natalia Scavone 8 , Karina Abreu iD , Jessy \\ Colman 10, Betania Medina 11 , Juan Irala 12 , Stefan Goertzen 13 , Vanesa \\ López ${ }^{14}$, Rosana Ortiz ${ }^{15}$, Graciela Lird 16 , Mario Martínez Mora iD 1
}

\footnotetext{
${ }^{1}$ Ministerio de Salud Pública y Bienestar Social, Laboratorio Central de Salud Pública. Av. Venezuela y Teniente Escurra, Asunción, Paraguay.

${ }^{2}$ Hospital del Trauma "Dr. Manuel Giani". Av. General Santos y Teodoro Mongelós, Asunción, Paraguay.

${ }^{3}$ Instituto de Previsión Social "Hospital Central", Av. Sacramento y Capitán Lombardo, Asunción, Paraguay.

${ }^{4}$ Ministerio de Salud Pública y Bienestar Social. Instituto de Medicina Tropical. Av. Venezuela y Florida, Asunción, Paraguay.

${ }^{5}$ Universidad Nacional del Este, Facultad de Ciencias de la Salud, Laboratorio Epidemiológico Regional de Alto Paraná. Calle Andreu Moleon. Minga Guazu, Paraguay.

${ }^{6}$ Hospital Universitario San Lorenzo. Ruta Mariscal Estigarribia. Km 15. San Lorenzo, Paraguay

${ }^{7}$ Sanatorio AMSA. Teniente Fariña y Capitán Figari, Asunción, Paraguay.

${ }^{8}$ Laboratorio Díaz Gill. Eligio Ayala 1384. Asunción, Paraguay.

${ }^{9}$ Meyer Lab. Coronel Irazábal y Mariscal Estigarribia, Asunción, Paraguay.

${ }^{10}$ Ministerio de Salud Pública y Bienestar Social. Hospital General Pediátrico "Niños de Acosta Ñu". Av. Arnaldo Bacigalupo, San Lorenzo, Paraguay.

${ }^{11}$ Hospital Policial Rigoberto Caballero. Mariscal López y J. Kubitschek. Asunción, Paraguay

${ }^{12}$ Laboratorio Analiza. Parapiti 1155. Asunción, Paraguay.

${ }^{13}$ Hospital Loma Plata. Loma Plata. Chaco Paraguayo, Paraguay.

${ }^{14}$ Ministerio de Salud Pública y Bienestar Social. Hospital Regional de Ciudad del Este. Ciudad del Este, Paraguay.

${ }^{15}$ Hospital General de Luque. Dora Gómez Bueno de Acuña. Luque, Paraguay.

${ }^{16}$ Universidad Nacional de Asunción, Facultad de Ciencias Médicas, Hospital de Clínicas. Avenida Mariscal López y Coronel Cazal, San Lorenzo, Paraguay.
}

Cómo referenciar este artículo/ How to reference this article:

\begin{abstract}
Melgarejo Touchet N, Busignani S, Dunjo $P$, Brítez M, Kawabata A, Silvagni M, et al. Primer reporte de Enterobacterales dobles productores de carbapenemasas en hospitales de Paraguay. Año 2021. Mem. Inst. Investig. Cienc. Salud. 2021; 19(3): $35-20$
\end{abstract}

\section{RE S U M E N}

tas carbapenemasas se encuentran ampllamente distribuldas en nuestro pals, tanto en bacilos gramnegativos fermentadores como no fermentadores. Durante 2021, se ha reportado incremento de cepas con estas enzimas. Con el objetivo de evaluar la doble producción de carbapenemasas en Enterobacterales y comunicar su circulación, fue puesta a punto una PCR convencional múltiple. Estudio retrospectivo en 128 aislamientos provenientes de 20 centros colaboradores de la Red Nacional de Vigilancia de la RAM (Capital, Central e interior del país), remitidos al LCSP entre febrero y setiembre de 2021, para confirmación y genotipificación de carbapenemasas. Se realizaron pruebas fenotípicas y colorimétricas con sustratos específicos, y pruebas genotípicas (PCR convencional múltiple) para la detección simultánea de varios genes de resistencia ( $b / a_{\mathrm{NDM}}, b / a_{\mathrm{KPC}}, b / a_{\mathrm{OXA}-48}-l i k e, b / a_{\mathrm{IMP}}$ y bla $\left.a_{\mathrm{VIM}}\right)$.

Fecha de recepción: octubre 2021. Fecha de aceptación: noviembre 2021

*Autor correspondiente: Dra Nancy Lorena Melgarejo Touchet. Laboratorio Central de Salud Pública. Av. Venezuela y Teniente Escurra, Asunción, Paraguay

Correo personal: nmtouchet@gmail.com Correo institucional: antimicrobiano.lcsp@mspbs.gov.py

Teléfono personal: +595981 173450 - Teléfono institucional: +59521292653 
De los 128 aislamientos estudiados, 107 correspondieron a Klebsiella pneumoniae, 14 a Enterobacter cloacae complex, entre otros; aislados en mayor frecuencia de muestras de orina $(30 \%)$, respiratorias $(30 \%)$, sangre y catéter $(24 \%)$. Los genes de resistencia a los carbapenemes detectados fueron: bla $a_{\mathrm{NDM}}(77,3 \%)$, bla $a_{\mathrm{KPC}}(17,2 \%)$; siendo confirmada la doble producción de carbapenemasas en 7 aislamientos $(5,5 \%)$ provenientes de 4 centros diferentes de la capital de país y uno de Central; 6 de ellas (K. pneumoniae) con bla $a_{\mathrm{NDM}}+$ bla $a_{\mathrm{KPC}}$ y 1 (E. cloacae complex) con bla $a_{\mathrm{NDM}}+$ bla $a_{\mathrm{OXA}-48}$-like; confirmando circulación de Enterobacterales dobles productores de carbapenemasas en el país (KPC+NDM y OXA+NDM); hallazgos que obligan a proveer de capacidades de detección, de manera a que se puedan tomar medidas oportunas y eficaces de contención y control.

Palabras clave: Resistencia antimicrobiana; carbapenemasa doble producción; bla $a_{\mathrm{NDM}} ;$ bla $\mathrm{KPC}_{1}$ bla $\mathrm{OXA}_{48}-$ like; Enterobacterales; Paraguay.

\title{
First report of double carbapenemases-producing Enterobacterales in hospitals of Paraguay. Year 2021.
}

\begin{abstract}
A B S T R A C T
Carbapenemases are widely distributed in our country, both in fermenting and nonfermenting gram-negative bacilli. During 2021, an increase in strains with these enzymes has been reported. In order to evaluate the double production of carbapenemases in Enterobacterales and communicate their circulation, a multiple conventional PCR was set up. Retrospective study carried out in 128 isolates from 20 collaborating centers of the National AMR Surveillance Network (Capital, Central and interior of the country), sent to the LCSP between February and September 2021, for confirmation and genotyping of carbapenemases. Phenotypic and colorimetric tests were performed with specific substrates, as well as genotypic tests (multiple conventional PCR) for the simultaneous detection of several resistance genes (bla $\mathrm{NDM}_{\text {, }}$ bla $_{\mathrm{KPC}}$, bla $\mathrm{OXA}_{\mathrm{O}-48-\mathrm{like}}, \mathrm{bla}_{\mathrm{IMP}}$ and $\left.\mathrm{bla}_{\mathrm{VIM}}\right)$. Of the 128 isolates studied, 107 corresponded to Klebsiella pneumoniae, 14 to Enterobacter cloacae complex, among others; isolated in higher frequency from urine $(30 \%)$, respiratory $(30 \%)$, blood and catheter $(24 \%)$ samples. The genes for resistance to carbapenems detected were: bla NDM $(77.3 \%)$, bla $_{\mathrm{KPC}}(17.2 \%)$; the double production of carbapenemases was confirmed in 7 isolates $(5.5 \%)$ from 4 different centers in the capital of the country and one in Central; 6 of them ( $K$. pneumoniae) with bla $\mathrm{NDM}+$ bla $_{\mathrm{KPC}}$ and 1 ( $E$. cloacae complex) with bla $\mathrm{NDM}+$

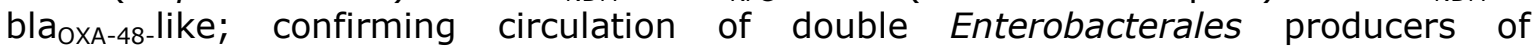
carbapenemases in the country (KPC + NDM and OXA + NDM); findings that require the provision of detection capabilities, so that timely and effective containment and control measures can be taken.
\end{abstract}

Keywords: Antimicrobial resistance, carbapenemase, double production, bla NDM, bla $_{\mathrm{KPC}}$, bla $\mathrm{OXA}_{\mathrm{A}}-48$-like, Enterobacterales, Paraguay.

\section{INTRODUCCIÓN}

La emergencia de Enterobacterales productoras de carbapenemasas constituye un enorme desafío para la salud pública, por el incremento en la morbi-mortalidad y los altos costos que generan el manejo y control de sus infecciones ${ }^{(1)}$.

Las principales carbapenemasas en este grupo bacteriano son: KPC (clase A), NDM, IMP, VIM (clase B) y OXA-48 (clase D) ${ }^{(2)}$; todas ellas de amplia distribución a nivel mundial, y endémicas en varios países de la región y el mundo.

En nuestro país, desde la confirmación de la carbapenemasa KPC en el año 2009 $(3,4)$ y la metalobetalactamasa NDM en el $2012^{(5)}$, varios centros asistenciales del país han reportado su circulación; sin embargo, hasta la fecha no ha sido reportada la doble producción de carbapenemasas en Enterobacterales ${ }^{(6-8)}$.

A fin de conocer acerca de la circulación de los gérmenes resistentes a los antimicrobianos y el genotipo de las enzimas involucradas, es fundamental el trabajo 
multidisciplinario que llevan a cabo los equipos de salud en la vigilancia de la resistencia a los antimicrobianos (RAM).

El Laboratorio Central de Salud Pública (LCSP), a través del Departamento de Bacteriología y Micología, coordina la Red Nacional de Vigilancia laboratorial de la Resistencia a los Antimicrobianos (RNVRAM) desde hace más de 20 años, trabajando de manera conjunta con los laboratorios públicos y privados de todo el país, en la caracterización molecular de las carbapenemasas circulantes.

Este año, los centros que conforman la RNVRAM han reportado incremento en el número de aislamiento de cepas de Enterobacterales resistentes a los carbapenemes, lo que motivó la puesta a punto de la técnica de PCR convencional múltiple para la detección simultanea de varios genes de carbapenemasas en este grupo bacteriano; los resultados de la implementación de la técnica confirmaron la circulación de Enterobacterales dobles productores de carbapenemasas en hospitales del país; constituyendo la comunicación de estos hallazgos el principal objetivo de la presente publicación.

\section{MATERIALES Y MÉTODO}

Estudio retrospectivo de corte transversal realizado con aislamientos de Enterobacterales provenientes de 20 centros colaboradores de la RNVRAM de Capital, Central e interior del país; remitidos al LCSP entre febrero y setiembre de 2021, para la confirmación de portación de carbapenemasas, y su genotipificación.

Los criterios de derivación de las cepas fueron:

- Halo de inhibición de imipenem $\leq 22 \mathrm{~mm}$, o

- Concentración inhibitoria mínima (CIM) en equipo automatizado (Vitek2 $\mathrm{C}^{\circledR}$ ) de imipenem $\geq 2 \mathrm{ug} / \mathrm{mL}^{(9)}$.

Fueron estudiados un total de 128 aislamientos remitidos, los cuales fueron sometidos a pruebas bioquímicas manuales para confirmación de especies bacterianas; tamizaje fenotípico de producción de carbapenemasas y estudios genotípicos para la detección de genes de resistencia a los carbepenemes.

Tamizaje fenotípico para detección de producción de carbapenemasas: Fue realizada por la prueba colorimétrica rápida blue carba ${ }^{(10)}$ y las pruebas de sinergias entre los discos de carbapenemes con los de ácido fenilborónico (APB) y ácido etilendiaminotetraacético/ácido mercaptoacético de sodio (EDTA/SMA). Además, de las pruebas de sinergia entre los discos de ceftazidima-avibactam (CZA, 14 ug) y EDTA (15 mm de distancia de centro a centro); $y$, entre los de aztreonam (AZT, 30 ug) y APB (10 mm de distancia de centro a centro).

Estudio genotípico para la detección de genes de resistencia: Realizado por reacción en cadena de la polimerasa (PCR) múltiple de punto final, utilizando cebadores específicos para la detección simultánea de los genes bla $a_{\mathrm{KPC}}, b / a_{\mathrm{NDM}}, b / a_{\mathrm{IMP}}$, blavim y bla OXA-48-like $^{(11)}$.

Para la obtención del ADN, se utilizó el método de lisis bacteriana por ebullición durante 10 minutos de una suspensión bacteriana de aproximadamente 0,5 Mac Farland en 300 uL de agua libre de RNAsa y centrifugada posteriormente a 10.000 rpm por 10 minutos Las reacciones de amplificación de los genes se realizaron en un termociclador TC-PRO (BOECO Germany) y los productos de amplificación se analizaron mediante electroforesis en gel de agarosa al $2 \%$ en tampón TAE buffer (PanReac AppliChem - ITW Reagents). Las imágenes de los patrones electroforéticos fueron obtenidas con el equipo fotodocumentador Gel Doc ${ }^{\mathrm{TM}}$ EZ Imager (BIO-RAD) y analizadas con el programa Image Lab 6.0 (BIO-RAD).

\section{RESULTADOS}

De los 128 aislamientos de Enterobacterales, 107 correspondieron a Klebsiella pneumoniae, 14 a Enterobacter cloacae complex, 2 a Escherichia coli, 2 a Citrobacter freundii, y 1 a Klebsiella oxytoca, Klebsiella aerogenes y Providencia stuartii. Los mismos fueron recuperados en mayor frecuencia de muestras de orina $(30 \%)$, respiratorias $(30 \%)$, sangre y catéter $(24 \%)$, secreciones $(13 \%)$, entre otras; de pacientes mayoritariamente hospitalizados (96\%).

Los genes de resistencia a los carbapenemes detectados fueron: 
- bla $\mathrm{NDM}_{\mathrm{N}}$ en 99 aislamientos $(77,3 \%)$, siendo predominante la especie bacteriana K. pneumoniae (88\%), seguida por E. cloacae complex $(6 \%)$;

- bla $\mathrm{KPC}_{\text {en }} 22$ aislamientos (17,2\%), prevalente en $K$. pneumoniae (64\%), seguida por E. cloacae complex (32\%);

- $b / a_{\mathrm{NDM}}+b l a_{\mathrm{KPC}}$ en 6 aislamientos, todos ellos de K. pneumoniae; $\mathrm{y}$,

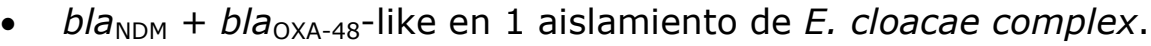

De los 7 aislamientos dobles productores de carbapenemasas $(5,5 \%) ; 6$ fueron provenientes de cuatro centros diferentes de la capital de país y uno de Central. Todas las cepas coproductoras de KPC+NDM correspondieron a K. pneumoniae, sin embargo, la coproducción de NDM + OXA-48-like fue confirmada en un aislamiento de $E$. cloacae complex. La Tabla 1 resume los resultados de caracterización molecular de las cepas estudiadas.

Tabla 1. Genotipificación de Enterobacterales productores de carbapenemasas por PCR convencional múltiple. Febrero a setiembre de 2021.Paraguay. N: 128.

\begin{tabular}{|c|c|c|}
\hline Genes detectados & $\begin{array}{l}\text { Número de } \\
\text { aislamientos }\end{array}$ & Cepa bacteriana \\
\hline$b^{\prime} a_{\mathrm{NDM}}$ & 99 & $\begin{array}{l}\text { K. pneumoniae (87) } \\
\text { E. cloacae complex }(6) \\
\text { E. coli }(2) \\
\text { C. freundii }(2) \\
\text { K. aerogenes }(1) \\
P . \text { stuartii }(1)\end{array}$ \\
\hline$b^{\prime} a_{\mathrm{KPC}}$ & 22 & $\begin{array}{l}\text { K. pneumoniae }(14) \\
\text { E. cloacae complex }(7) \\
\text { K. oxytoca }(1)\end{array}$ \\
\hline$b / a_{\mathrm{NDM}}+b / a_{\mathrm{KPC}}$ & 6 & K. pneumoniae (6) \\
\hline$b^{\prime} / a_{\mathrm{NDM}}+b / a_{\mathrm{OXA}-48}$-like & 1 & E. cloacae complex (1) \\
\hline
\end{tabular}

Fuente: Dpto. Bacteriología y Micología, Laboratorio Central de Salud Pública. MSPyBS. Paraguay.

En cuanto a las características de los aislamientos con doble producción de carbapenemasas y sus orígenes; en el $100 \%$ fueron recuperados de muestras clínicas tomadas de pacientes hospitalizados con más de 25 días de internación. La Tabla 2 resume las características más importantes proveídas por los centros remitentes.

Tabla 2. Características de los aislamientos dobles productores de carbapenemasas.

Paraguay. Año 2021.

\begin{tabular}{|c|c|c|c|c|c|c|}
\hline Paciente & $\begin{array}{l}\text { Centro de } \\
\text { internación }\end{array}$ & $\begin{array}{c}\text { Edad } \\
\text { (años) } \\
\text { sexo }\end{array}$ & $\begin{array}{l}\text { Patología } \\
\text { de base }\end{array}$ & $\begin{array}{l}\text { Muestra } \\
\text { clínica }\end{array}$ & $\begin{array}{l}\text { Aislamiento } \\
\text { bacteriano }\end{array}$ & Desenlace \\
\hline 1 & Centro A & $\begin{array}{c}78 \\
F\end{array}$ & $\begin{array}{l}\text { - Hipertensión } \\
\text { arterial } \\
\text { - Cardiopatía } \\
\text { isquémica } \\
\text { - Diabetes } \\
\text { - Hipotiroidismo } \\
\text { - Trastorno } \\
\\
\text { depresivo }\end{array}$ & re & $\begin{array}{l}\text { kpn } \\
\text { NDM+KPC }\end{array}$ & Óbito \\
\hline 2 & Centro B & $\begin{array}{c}61 \\
F\end{array}$ & $\begin{array}{l}\text { - Complicaciones } \\
\text { post-covid }\end{array}$ & se & $\begin{array}{l}\mathrm{kpn} \\
\mathrm{NDM}+\mathrm{KPC}\end{array}$ & Óbito \\
\hline 3 & Centro C & $\begin{array}{l}59 \\
M\end{array}$ & $\begin{array}{l}\text { Neumonía } \\
\text { asociada a } \\
\text { SARS-CoV-2 }\end{array}$ & es & $\begin{array}{l}\mathrm{kpn} \\
\mathrm{NDM}+\mathrm{KPC}\end{array}$ & Alta médica \\
\hline
\end{tabular}




\begin{tabular}{|c|c|c|c|c|c|c|}
\hline 4 & Centro D & $\begin{array}{l}35 \\
M\end{array}$ & $\begin{array}{l}\text { - Traumatismo } \\
\text { por accidente de } \\
\text { tránsito } \\
\text { - Sin patología de } \\
\text { base }\end{array}$ & ao & $\begin{array}{l}\text { kpn } \\
\text { NDM+KPC }\end{array}$ & Alta medica \\
\hline 5 & Centro E & $\begin{array}{l}57 \\
M\end{array}$ & - Tumor rectal & $\mathrm{ca}$ & $\begin{array}{l}\text { ecl } \\
\text { NDM+OXA- } \\
48 \text {-like }\end{array}$ & Óbito \\
\hline 6 & Centro D & $\begin{array}{l}47 \\
M\end{array}$ & $\begin{array}{l}\text { - Traumatismo } \\
\text { por accidente de } \\
\text { tránsito } \\
\text { - Sin patología de } \\
\text { base }\end{array}$ & $\begin{array}{l}\mathrm{sa} \\
\mathrm{ca}\end{array}$ & $\begin{array}{l}\text { Kpn } \\
\text { NDM+KPC }\end{array}$ & $\begin{array}{l}\text { Sigue en } \\
\text { internación }\end{array}$ \\
\hline 7 & Centro D & $\begin{array}{l}24 \\
M\end{array}$ & $\begin{array}{l}\text { - Traumatismo } \\
\text { por accidente de } \\
\text { tránsito } \\
\text { - Sin patología de } \\
\text { base }\end{array}$ & $\mathrm{ca}$ & $\begin{array}{l}\mathrm{kpn} \\
\mathrm{NDM}+\mathrm{KPC}\end{array}$ & $\begin{array}{l}\text { Sigue en } \\
\text { internación }\end{array}$ \\
\hline
\end{tabular}

Fuente: Dpto. Bacteriología y Micología, Laboratorio Central de Salud Pública. MSPyBS. Paraguay.

Los resultados de los estudios fenotípicos de las cepas dobles productoras de carbapenemasas fueron:

- K. pneumoniae (6):

- Blue carba test: Positivo

- Sinergia entre disco de CZA (14 ug) y disco de EDTA: Positivo.

- Sinergia entre discos de AZT (30 ug) - discos de APB: Positivo.

- E. cloacae complex (1):

- Blue carba test: Positivo

- Sinergia entre disco de CZA (14 ug) y disco de EDTA: Positivo.

- Sinergia entre disco de AZT (30 ug) y disco de APB: Negativo.

La Figura 1 muestra los resultados de sinergias de las cepas de $K$. pneumoniae dobles productoras de carbapenemasas; y la Figura 2 muestra los resultados de caracterización molecular llevadas a cabo por PCR convencional múltiple de las cepas estudiadas con los respetivos controles de calidad.

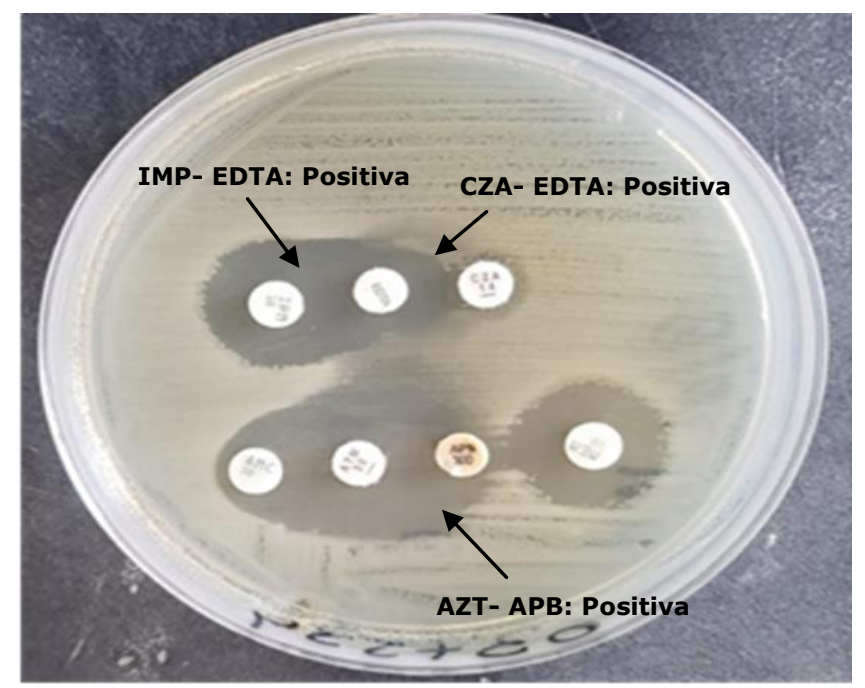

CZA: ceftazidima/avibactam; IMP: imipenem; AZT: aztreonam; MEM; meropenem; APB: ácido fenilborónico.

Figura 1. Pruebas de sinergia realizadas en aislamientos de $K$. pneumoniae (NDM+KPC). 


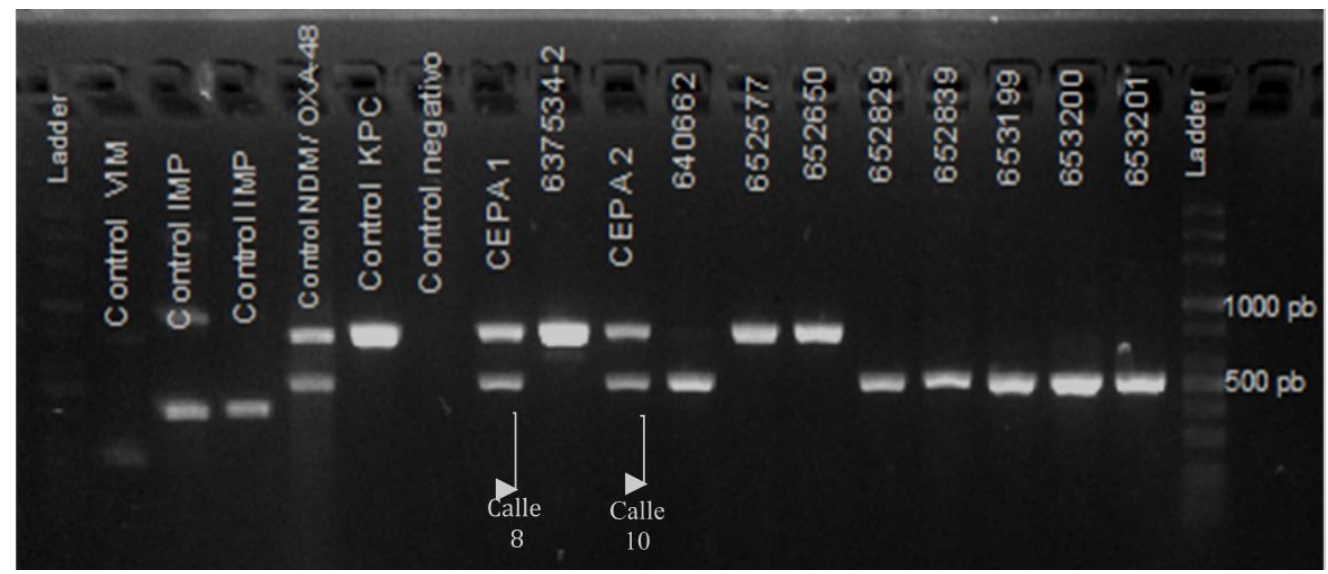

Las cepas 1 y 2 (calles 8 y 10); corresponden a $K$. pneumoniae con doble producción de genes de carbapenemasas (bla $a_{\mathrm{KPC}}$ y bla $a_{\mathrm{NDM}}$ ).

Figura 2. PCR convencional múltiple para detección simultánea de genes de resistencia a carbepenemes. Paraguay. N: 128. Año 2021.

\section{CONCLUSIÓN Y DISCUSIÓN}

Este es el primer reporte en el país de detección en Enterobacterales con doble producción de carbapenemasas, con portación de genes $b / a_{\mathrm{NDM}}+b / a_{\mathrm{KPC}}$ en aislamientos de K. pneumoniae; y de genes $b / a_{\mathrm{NDM}}+$ bla $_{\mathrm{OXA}-48}$-like en $E$. cloacae complex. Hasta la fecha, solo hemos confirmado las producciones de carbapenemasas KPC y NDM en Enterobacterales, siendo éste el primer hallazgo de OXA-48; una enzima ya descrita en países de la región (Argentina, Brasil y Colombia) ${ }^{12-14}$, y una de las más frecuentes en países de $\operatorname{Europa}^{(15,16)}$.

Los primeros hallazgos de coproducción de carbapenemasas en Enterobacterales fueron confirmados en cepas provenientes de la India: Karthikeyan K y colaboradores informaron en el año 2010 el primer reporte de coexistencia de OXA-48 y NDM-1 ${ }^{(17)}$, y en el año 2012, Kumarasamy y colaboradores publicaron la coproducción de NDM-1 y KPC-2 en aislamiento de $K$. pneumoniae recuperado de una muestra de orina en julio de $2010^{(18)}$. A partir de entonces, varios países del mundo han confirmado hallazgos similares de coproducción de diferentes genotipos ${ }^{(19-21)}$.

En países de la región, los primeros en reportar coproducción de carbapenemasas fueron investigadores de Brasil: Nava RG y colaboradores en el $2019^{(22)}$; y, Oliveira EM y colaboradores en el $2020^{(23)}$, describiendo la portación de genes bla ${ }_{\mathrm{NDM}}+$ bla $\mathrm{KPC}$ en aislamientos de $K$. pneumoniae.

En abril del 2021 el Laboratorio de Referencia Regional "Dr. Carlos Malbrán". INEI. ANLIS, emitió una Alerta Epidemiológica: Emergencia de dobles productores de carbapenemasa KPC+MBL", en la que se describe la confirmación en la primera ola de la pandemia por COVID-19 la emergencia y diseminación de colonización/infección de Enterobacterales productores de combinaciones de carbapenemasas en la Argentina, de los genotipos KPC+NDM, NDM+OXA-163 y NDM+IMP ${ }^{(24)}$.

A raíz de los reportes regionales, el Laboratorio de Referencia Nacional llevó a cabo la estandarización de metodologías (fenotípicas y genotípicas) y estudios retrospectivos en los aislamientos productores de carbapenemasas, y los resultados de los hallazgos fueron socializados en un comunicado en setiembre del presente año, con las recomendaciones para los laboratorios de microbiología (25). La alerta epidemiológica regional por producción de dobles carbapenemasas fue emitida por la Organización Panamericana de la Salud el 22 de octubre ${ }^{(26)}$ y la alerta epidemiológica nacional por el Ministerio de Salud Pública y Bienestar Social el 2 de noviembre de $2021^{(27)}$.

Esta emergencia y diseminación de bacterias coproductoras de carbapenemasas, requiere una respuesta firme, coordinada y protocolizada por parte de todo el equipo 
sanitario multidisciplinario y autoridades de salud humana, veterinaria y ambiental, que permitan el control precoz y minimice su impacto negativo.

Nuestros hallazgos además obligan a proveer capacidades de detección a los laboratorios de bacteriología de todo el país, de manera a que se puedan tomar medidas oportunas y eficaces de contención y control.

CONFLICTO DE INTERES: No declarado.

FINANCIAMIENTO: Ninguno.

\section{CONTRIBUCIÓN DE AUTORES}

Melgarejo Touchet Nancy: Autor principal. Idea, Elaboración del Protocolo, recolección y análisis de los datos, presentación de resultados, redacción del manuscrito.

Martínez Mario: Asesoría, revisión crítica del manuscrito.

Busignani Sofía: Estudios fenotípicos y genotípicos completos de los aislamientos bajo investigación.

Pamela Dunjo: Estudios fenotípicos y genotípicos completos de los aislamientos bajo investigación.

Mariel Brítez: Apoyo en estudios genotípicos de los aislamientos bajo investigación. Aníbal Kawabata, Marlene Silvagni, Carolina Rojas, Eva Mereles, Evelyn López, Juana Salinas, Natalia Scavone, Karina Abreu, Jessy Colman, Betania Medina, Juan Irala, Stefan Goertzen, Vanesa López, Rosana Ortiz, Graciela Lird: Detección fenotípica de resistencias inusuales en las cepas de interés; y remisión de estas al Laboratorio de Referencia.

\section{REFERENCIAS BIBLIOGRÁFICAS}

1. Pujol M, Limón E. Epidemiología general de las infecciones nosocomiales. Sistemas y programas de vigilancia [General epidemiology of nosocomial infections. Surveillance systems and programs]. Enferm Infecc Microbiol Clin. 2013 Feb; 31(2): 108-13. Doi: 10.1016/j.eimc.2013.01.001. PMID: 23357654.

2. Queenan AM, Bush K. Carbapenemases: the versatile beta-lactamases. Clin Microbiol Rev. 2007 Jul; 20(3): 440-58, table of contents.

Doi: $10.1128 / \mathrm{cmr} .00001-07$ PMID: 17630334; PMCID: PMC1932750.

3. Melgarejo N, Martínez M, Franco R, Lird G, Laconich M, Aguilar G. Detección de carbapenemasa (KPC) en Enterobacter en un hospital de Asunción, Paraguay. VII Congreso paraguayo de Infectología. I Jornada de Microbiología Clínica. V Jornada de Enfermería. 6, 7, 8 de noviembre de 2009. Asunción, Paraguay.

4. Melgarejo N, Martínez M, Franco R, Falcón M. Enterobacterias resistentes a Carbapenemes por producción de KPC, aisladas en hospitales de Asunción y Departamento Central. Revista de Salud Pública del Paraguay. 2013; 3(1): 30-5. Disponible en: https://fiadmin.bvsalud.org/document/view/w9y hv

5. Dirección General de Vigilancia de la Salud. Ministerio de Salud Pública y
Bienestar Social. Alerta Epidemiológica. Primer hallazgo de Metalobetalactamasa. New Delhi (NDM) en Paraguay. 26 de noviembre de 2012. Disponible en:

https://www.mspbs.gov.py/dependenci as/imt/uploads/Documento/alerta6.pdf.

6. Melgarejo $N$, Martínez $M$, Franco $R$, Falcón M. Enterobacterias resistentes a Carbapenemes por producción de KPC, aisladas en hospitales de Asunción y Departamento Central. Rev. Salud Pública Parag. 2013 3(1): 30-5. Disponible en: https://fiadmin.bvsalud.org/document/view/w9y hv.

7. Rivas $M$, Ortiz $H$, Almada $P$, Arguello $R$, Melgarejo N, Martínez C. Escherichia coli metalobetalactamasa en un hospital de alta complejidad en Paraguay. Rev. virtual Soc. Parag. Med. Int 2016; 3 (2):120-3. Disponible en: http://scielo.iics.una.py/scielo.php?scrip $\mathrm{t}=\mathrm{sci}$ arttext\&pid $=\mathrm{S} 2312-$ $38932016000200011 \& \operatorname{lng}=e n \% 22 \operatorname{lng}=\mathrm{e}$ n.

8. Melgarejo-Touchet $\mathrm{N}$, Brítez $\mathrm{CM}$, Busignani $S$, Falcón $M$, López $E$, Laconich M, et al. (2021). Caracterización molecular de carbapenemasas en bacilos gramnegativos circulantes en hospitales de Paraguay. Primer cuatrimestre 2021. Memorias del Instituto de 
Investigaciones en Ciencias de la Salud, 2021; 19(2): 49-58. Doi:

$10.18004 / \mathrm{mem} . \mathrm{iics} / 1812$

9528/2021.019.02.49.

9. Servicio Antimicrobianos, Instituto Nacional de Enfermedades Infecciosas, Administración Nacional de Laboratorios e Institutos de Salud "Dr. Carlos G. Malbrán". Criterios para sospecha de carbapenemasas en Enterobacterias 2019. Disponible en:

http://antimicrobianos.com.ar/ATB/wpcontent/uploads/2019/10/CRITERIOS-

PARA-SOSPECHA-DE-

CARBAPENEMASAS-EN-

ENTEROBACTERIAS-2019.pdf.

10. Pires J, Novais A, Peixe L. Blue-carba, an easy biochemical test for detection of diverse carbapenemase producers directly from bacterial cultures. J Clin Microbiol. 2013 Dec; 51(12): 4281-3. Doi: $10.1128 / \mathrm{jcm} .01634-13$

Epub 2013 Oct 9. PMID: 24108615; PMCID: PMC3838089.

11. Servicio Antimicrobianos, Instituto Nacional de Enfermedades Infecciosas, Administración Nacional de Laboratorios e Institutos de Salud "Dr. Carlos G. Malbrán". Protocolo de PCR multiplex para detección de carbapenemasas. Disponible en:

http://antimicrobianos.com.ar/ATB/wpcontent/uploads/2021/01/DeteccionCBP-Multiplex.pdf

12. Ocampo AM, Chen $L$, Cienfuegos AV, Roncancio G, Chavda KD, Kreiswirth BN, Jiménez JN. A Two-Year Surveillance in Five Colombian Tertiary Care Hospitals Reveals High Frequency of Non-CG258 Clones of Carbapenem-Resistant Klebsiella pneumoniae with Distinct Clinical Characteristics. Antimicrob Agents Chemother. 2015; 60(1): 33242. Doi: 10.1128/aac.01775-15

13. Pereira $\mathrm{PS}$, Borghi $\mathrm{M}$, de Araújo $C F$, Aires CA, Oliveira JC, Asensi MD, Carvalho-Assef AP. Clonal Dissemination of OXA-370-Producing Klebsiella pneumoniae in Rio de Janeiro, Brazil. Antimicrob Agents Chemother. 2015; 59 (8): 4453-6. Doi: 10.1128/AAC.04243-14

14. Andrés $P$, Rodríguez $A$, Albornoz $E$, Rapoport M, Saa G, Pasterán F, Corso A, Fernández A. Emergencia de OXA370, una variante de la carbapenemasa OXA-48, en un aislamiento clínico del Complejo Enterobacter cloacae en Argentina. XV Congreso Argentino de Microbiología (CAM 2019). Poster 008. Disponible en:

http://antimicrobianos.com.ar/ATB/wpcontent/uploads/2019/09/EMERGENCIA
-DE-OXA370-UNA-VARIANTE-DE-LACARBAPENEMASA-OXA48.pdf

15. Van Duin D, Doi $Y$. The global epidemiology of carbapenemaseproducing Enterobacteriaceae. Virulence. 2017; 8(4): 460- 9. doi: 10.1080/21505594.2016.1222343

16. Galan-Sánchez F, Ruiz del Castillo B, Marín-Casanova $\mathrm{P}$, Rodríguez-Iglesias M. Caracterización de blaOXA-48 detectada en cepas clínicas de Enterobacter cloacae aisladas en el sur de España [Characterization of blaOXA48 in Enterobacter cloacae clinical strains in southern Spain]. Enferm Infecc Microbiol Clin. 2012 Nov; 30(9): 584-5. Spanish. doi: 10.1016/j.eimc.2012.05.002. Epub 2012 Jun 21. PMID: 22727535.

17. Karthikeyan K, Toleman M, Giske CG. First report of the co-existence of blaOXA-48 or blaOXA-48-like gene with blaNDM-1 in Enterobacteriaceae from India. Clin Microbiol Infect 2010; 16 Suppl 2: S187.

18. Kumarasamy K, Kalyanasundaram A. Emergence of Klebsiella pneumoniae isolate co-producing NDM-1 with KPC-2 from India. J Antimicrob Chemother. 2012 Jan; 67(1):243-4. doi: 10.1093/jac/dkr431. Epub 2011 Oct 11. PMID: 21990048.

19. Loqman S, Soraa N, Diene SM, Rolain JM. Dissemination of Carbapenemases (OXA-48, NDM and VIM) Producing Enterobacteriaceae Isolated from the Mohamed VI University Hospital in Marrakech, Morocco. Antibiotics (Basel). 2021 Apr 26; 10(5): 492. doi: 10.3390/antibiotics10050492. PMID: 33925772; PMCID: PMC8145435.

20. Thomson GK, Snyder JW, McElheny CL, Thomson KS, Doi Y. Coproduction of KPC-18 and VIM-1 Carbapenemases by Enterobacter cloacae: Implications for Newer $\beta$-Lactam- $\beta$-Lactamase Inhibitor Combinations. J Clin Microbiol. 2016 Mar; 54(3):791-4. Doi: 10.1128/JCM.02739-15. Epub 2015 Dec 30. PMID: 26719440; PMCID: PMC4767958.

21. Poirel L, Ros A, Carricajo A, Berthelot $P$, Pozzetto B, Bernabeu S, Nordmann P. Extremely drug-resistant Citrobacter freundii isolate producing NDM-1 and other carbapenemases identified in a patient returning from India. Antimicrob Agents Chemother. 2011 Jan; 55(1):447-8. doi: 10.1128/AAC.0130510. Epub 2010 Oct 25. PMID: 20974865; PMCID: PMC3019622.

22. Nava RG, Oliveira-Silva $M$, NakamuraSilva R, Pitondo-Silva A, Vespero EC. New sequence type in multidrug- 
resistant Klebsiella pneumoniae harboring the blaNDM-1-encoding gene in Brazil. Int J Infect Dis. 2019 Feb; 79: 101-3. doi: 10.1016/j.ijid.2018.11.012. Epub 2018 Nov 26. PMID: 30496852.

23. Oliveira ÉM, Beltrão EMB, Scavuzzi AML, Barros JF, Lopes ACS. High plasmid variability, and the presence of IncFIB, IncQ, IncA/C, IncHI1B, and IncL/M in clinical isolates of Klebsiella pneumoniae with bla KPC and bla NDM from patients at a public hospital in Brazil. Rev Soc Bras Med Trop. 2020; 53: e20200397. doi: 10.1590/0037-8682-0397-2020.

24. Programa Nacional de Control de Calidad en Bacteriología INEI-ANLIS "Dr. Carlos G. Malbrán". Alerta Epidemiológica: Emergencia de Enterobacterales dobles productores de carbapenemasas. INEI. ANLIS. Malbrán. 2021. Disponible en: http://antimicrobianos.com.ar/2021/?ca $\underline{\mathrm{t}=37}$.

25. Melgarejo N, Falcón M, Busignani S, Britez $M$, Dunjo P. Detección de Enterobacterales dobles productores de carbapenemasas en hospitales de Paraguay. Revista del Nacional 2021; 13 (1) suplemento. Disponible en http://www.revistadelnacional.com.py/i ndex.php/inicio/Suplemento.

26. Organización Panamericana de la Salud. Alerta epidemiológica. Emergencia e incremento de nuevas combinaciones de carbapenemasas en Enterobacterales en Latinoamérica y el Caribe. 22 de octubre de 2021. Disponible en https://www.paho.org/es/documentos/a lerta-epidemiologica-emergencia-eincremento-nuevas-combinacionescarbapenemasas.

27. Ministerio de Salud Pública y Bienestar Social. Alerta epidemiológica $\mathrm{N}^{\circ}$ 2/2021. Detección de Enterobacterias multirresistentes y con doble portación de carbapenemasas. Disponible en http://dgvs.mspbs.gov.py/files/slider/B OLETIN DETECCION\%20DE\%20ENTER OBACTERIAS.pdf. 